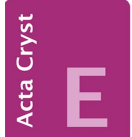
COMMUNICATIONS

ISSN 2056-9890

\section{Crystal structure of 2,2-dimethyl-N- (pyridin-3-yl)propanamide}

\author{
Gamal A. El-Hiti, ${ }^{\text {** Keith Smith, }}{ }^{\text {a }}$ Amany S. Hegazy, \\ Saud A. Alanazi ${ }^{\mathrm{a}}$ and Benson M. Kariuki ${ }^{\mathrm{b}}$ * \\ ${ }^{a}$ Cornea Research Chair, Department of Optometry, College of Applied Medical \\ Sciences, King Saud University, PO Box 10219, Riyadh 11433, Saudi Arabia, and

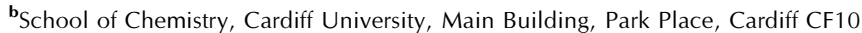 \\ 3AT, Wales. *Correspondence e-mail: gelhiti@ksu.edu.sa, kariukib@cardiff.ac.uk
}

Received 6 March 2015; accepted 14 March 2015

Edited by H. Stoeckli-Evans, University of Neuchâtel, Switzerland

In the title compound, $\mathrm{C}_{10} \mathrm{H}_{14} \mathrm{~N}_{2} \mathrm{O}$, the pyridine ring is inclined to the mean plane of the amide moiety $[\mathrm{N}-\mathrm{C}(=\mathrm{O}) \mathrm{C}]$ by $17.60(8)^{\circ}$. There is an intramolecular $\mathrm{C}-\mathrm{H} \cdots \mathrm{O}$ hydrogen bond present involving the carbonyl $\mathrm{O}$ atom. In the crystal, molecules are linked via $\mathrm{N}-\mathrm{H} \cdots \mathrm{N}$ hydrogen bonds, forming chains propagating along [100]. The tert-butyl group is disordered over two sets of sites with a refined occupancy ratio of 0.758 (12):0.242 (12).

Keywords: crystal structure; pyridine; propanamide; $\mathrm{N}-\mathrm{H} \cdots \mathrm{N}$ hydrogen bonds.

CCDC reference: 1054113

\section{Related literature}

For related biologically active pyridine derivatives, see: de Candia et al. (2013); Thorat et al. (2013); Abdel-Megeed et al. (2012). For pyridine ring-system modifications, see: El-Hiti et al. (2015); Smith et al. (2012, 2013); Londregan et al. (2009); Joule \& Mills (2000); Turner (1983). For the crystal structures of related compounds, see: El-Hiti et al. (2014); Seidler et al. (2011); Koch et al. (2008); Mazik et al. (2004).

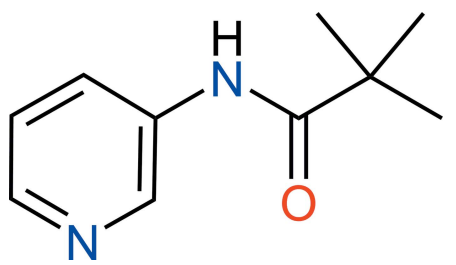

\section{Experimental}

2.1. Crystal data

$\mathrm{C}_{10} \mathrm{H}_{14} \mathrm{~N}_{2} \mathrm{O}$

$M_{r}=178.23$

Orthorhombic, $\mathrm{Pbca}$

$a=11.2453(3) \AA$

$b=10.5272(3) \AA$

$c=17.5339(6) \AA$

$V=2075.69(11) \AA^{3}$

$Z=8$

$\mathrm{Cu} K \alpha$ radiation

$\mu=0.60 \mathrm{~mm}^{-1}$

$T=293 \mathrm{~K}$

$0.23 \times 0.19 \times 0.06 \mathrm{~mm}$

\subsection{Data collection}

Agilent SuperNova (Dual, $\mathrm{Cu}$ at zero, Atlas) diffractometer Absorption correction: multi-scan (CrysAlis PRO; Agilent, 2014) $T_{\min }=0.840, T_{\max }=1.000$

7164 measured reflections 2065 independent reflections 1722 reflections with $I>2 \sigma(I)$ $R_{\text {int }}=0.017$

\subsection{Refinement}

$R\left[F^{2}>2 \sigma\left(F^{2}\right)\right]=0.041$

$w R\left(F^{2}\right)=0.127$

$S=1.05$

2065 reflections

153 parameters

114 restraints

$\mathrm{H}$-atom parameters constrained

$\Delta \rho_{\max }=0.19 \mathrm{e} \AA^{-3}$

$\Delta \rho_{\min }=-0.16$ e $\AA^{-3}$

Table 1

Hydrogen-bond geometry $\left(\AA{ }^{\circ}\right)$.

\begin{tabular}{lllll}
\hline$D-\mathrm{H} \cdots A$ & $D-\mathrm{H}$ & $\mathrm{H} \cdots A$ & $D \cdots A$ & $D-\mathrm{H} \cdots A$ \\
\hline $\mathrm{C} 2-\mathrm{H} 2 \cdots \mathrm{O} 1$ & 0.93 & 2.25 & $2.8263(18)$ & 119 \\
$\mathrm{~N} 1-\mathrm{H} 1 \cdots \mathrm{N}{ }^{\mathrm{i}}$ & 0.86 & 2.17 & $3.0012(15)$ & 164 \\
\hline
\end{tabular}

Symmetry code: (i) $x+\frac{1}{2}, y,-z+\frac{1}{2}$.

Data collection: CrysAlis PRO (Agilent, 2014); cell refinement: CrysAlis PRO; data reduction: CrysAlis PRO; program(s) used to solve structure: SHELXS2013 (Sheldrick, 2008); program(s) used to refine structure: SHELXL2013 (Sheldrick, 2015); molecular graphics: ORTEP-3 for Windows (Farrugia, 2012); software used to prepare material for publication: Win $G X$ (Farrugia, 2012).

\title{
Acknowledgements
}

The authors extend their appreciation to the Cornea Research Chair, Department of Optometry, College of Applied Medical Sciences, King Saud University, for funding this research, and to Cardiff University for continued support.

Supporting information for this paper is available from the IUCr electronic archives (Reference: SU5094).

\section{References}

Abdel-Megeed, M. F., Badr, B. E., Azaam, M. M. \& El-Hiti, G. A. (2012). Bioorg. Med. Chem. 20, 2252-2258.

Agilent (2014). CrysAlis PRO. Agilent Technologies, Yarnton, England.

Candia, M. de, Fiorella, F., Lopopolo, G., Carotti, A., Romano, M. R., Lograno, M. D., Martel, S., Carrupt, P.-A., Belviso, B. D., Caliandro, R. \& Altomare, C. (2013). J. Med. Chem. 56, 8696-8711.

El-Hiti, G. A., Smith, K., Balakit, A. A., Hegazy, A. S. \& Kariuki, B. M. (2014). Acta Cryst. E70, o351-o352.

El-Hiti, G. A., Smith, K. \& Hegazy, A. S. (2015). Heterocycles, 91, 479-504. 
Farrugia, L. J. (2012). J. Appl. Cryst. 45, 849-854.

Joule, J. A. \& Mills, K. (2000). Heterocycl. Chem. 4th ed. England: Blackwell Science Publishers.

Koch, P., Schollmeyer, D. \& Laufer, S. (2008). Acta Cryst. E64, o2216.

Londregan, A. T., Storer, G., Wooten, C., Yang, X. \& Warmus, J. (2009). Tetrahedron Lett. 50, 1986-1988.

Mazik, M., Radunz, W. \& Boese, R. (2004). J. Org. Chem. 69, 7448-7462.

Seidler, T., Gryl, M., Trzewik, B. \& Stadnicka, K. (2011). Acta Cryst. E67, o1507.

Sheldrick, G. M. (2008). Acta Cryst. A64, 112-122.
Sheldrick, G. M. (2015). Acta Cryst. C71, 3-8.

Smith, K., El-Hiti, G. A., Alshammari, M. B. \& Fekri, A. (2013). Synthesis, 45, 3426-3434.

Smith, K., El-Hiti, G. A., Fekri, A. \& Alshammari, M. B. (2012). Heterocycles, 86, 391-410.

Thorat, S. A., Kang, D. W., Ryu, H. C., Kim, H. S., Kim, H. S., Ann, J., Ha, T. Kim, S.-E., Son, K., Choi, S., Blumberg, P. M., Frank, R., Bahrenberg, G., Schiene, K., Christoph, T. \& Lee, J. (2013). Eur. J. Med. Chem. 64, 589-602. Turner, J. A. (1983). J. Org. Chem. 48, 3401-3408. 


\title{
supporting information
}

Acta Cryst. (2015). E71, o246-o247 [doi:10.1107/S2056989015005289]

\section{Crystal structure of 2,2-dimethyl-N-(pyridin-3-yl) propanamide}

\author{
Gamal A. El-Hiti, Keith Smith, Amany S. Hegazy, Saud A. Alanazi and Benson M. Kariuki
}

\section{S1. Introduction}

Pyridine derivatives are interesting compounds (Joule \& Mills, 2000) since they show a range of biological activities (Thorat et al., 2013) such as anticoagulant (de Candia et al., 2013) and antimicrobial (Abdel-Megeed et al., 2012) properties. Various simple and efficient processes have been developed for modification of the pyridine ring system (ElHiti et al., 2015; Smith et al., 2013, Smith et al., 2012, Londregan et al., 2009; Turner, 1983). The X-ray crystal structures of related compounds have been reported (El-Hiti et al., 2014; Seidler et al., 2011; Koch et al., 2008; Mazik et al., 2004).

\section{S2. Experimental}

The title compound was obtained in $73 \%$ yield from the reaction of 3-aminopyridine with pivaloyl chloride in the presence of triethylamine in dichloromethane at $273 \mathrm{~K}$ for $15 \mathrm{~min}$ and then at room temperature for $2 \mathrm{~h}$ (Turner, 1983). Crystallization from a mixture of ethyl acetate and hexane gave colourless crystals of the title compound. The spectroscopic and analytical data for the title compound were identical with those reported previously (Turner, 1983)

\section{S2.1. Refinement}

The $\mathrm{N}$ - and $\mathrm{C}$-bound $\mathrm{H}$ atoms were included in calculated positions and refined as riding: $\mathrm{N}-\mathrm{H}=0.86 \AA, \mathrm{C}-\mathrm{H}=0.93$ $0.98 \AA$ with $\mathrm{U}_{\mathrm{iso}}(\mathrm{H})=1.5 \mathrm{U}_{\mathrm{eq}}(\mathrm{C})$ for methyl $\mathrm{H}$ atoms and $=1.2 \mathrm{U}_{\mathrm{eq}}(\mathrm{C})$ for other $\mathrm{H}$ atoms. The $t$-butyl group is disordered over two sites and was refined with bond length constraints to give a refined occupancy ratio of 0.758 (12):0.242 (12).

\section{S3. Results and discussion}

The molecular structure of the title compound is illustrated in Fig. 1. The pyridine ring is inclined to the mean plane of the amide moiety $[\mathrm{N} 1-\mathrm{C} 6(=\mathrm{O} 1)-\mathrm{C} 7]$ by $17.60(8)^{\circ}$. There is an intramolecular $\mathrm{C}-\mathrm{H} \cdots \mathrm{O}$ hydrogen bond present involving the carbonyl $\mathrm{O}$ atom (Table 1).

In the crystal, molecules are linked via $\mathrm{N}-\mathrm{H} \cdots \mathrm{N}$ hydrogen bonds forming chains propagating along [100]; see Table 1 and Fig. 2. 


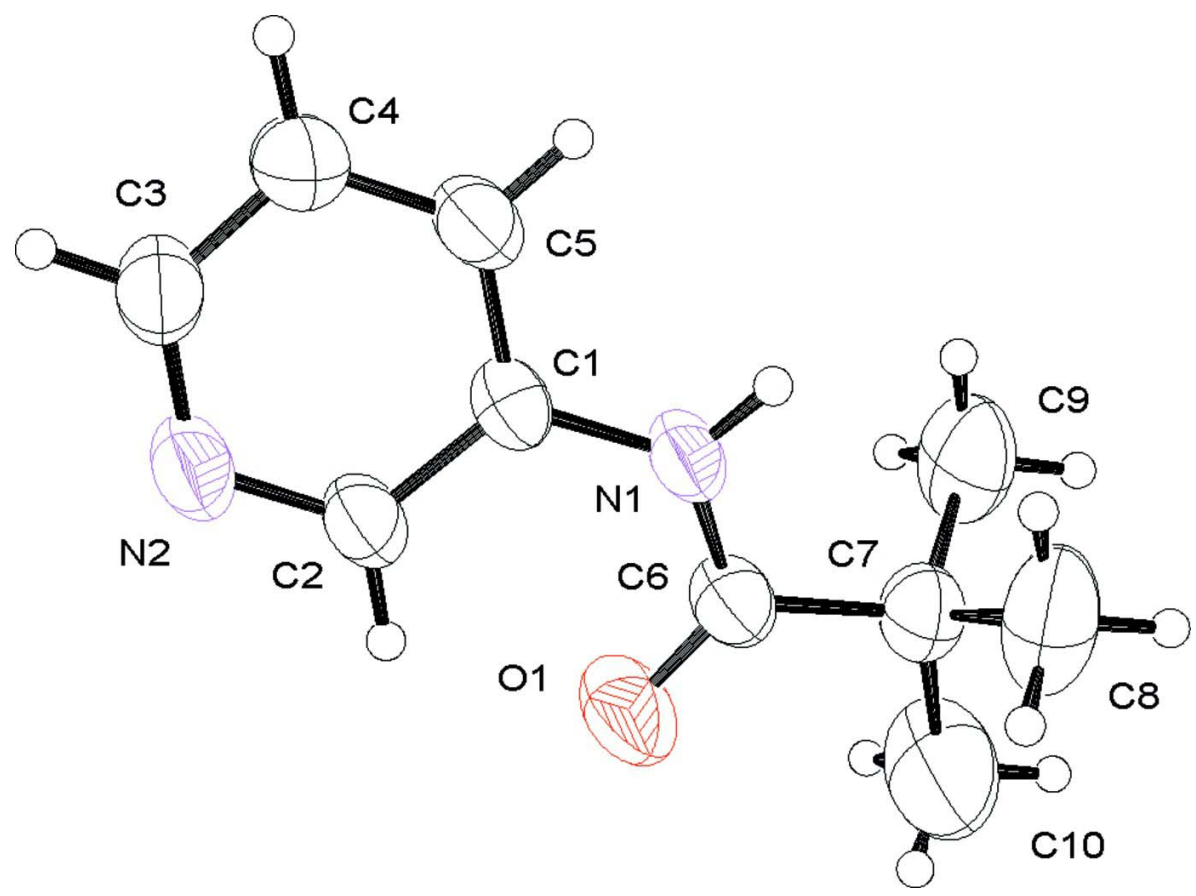

Figure 1

The molecular structure of the title compound, with atom labelling. Displacement ellipsoids are drawn at the 50\% probability level. Only the major component of the disordered $t$-butyl group is shown. 


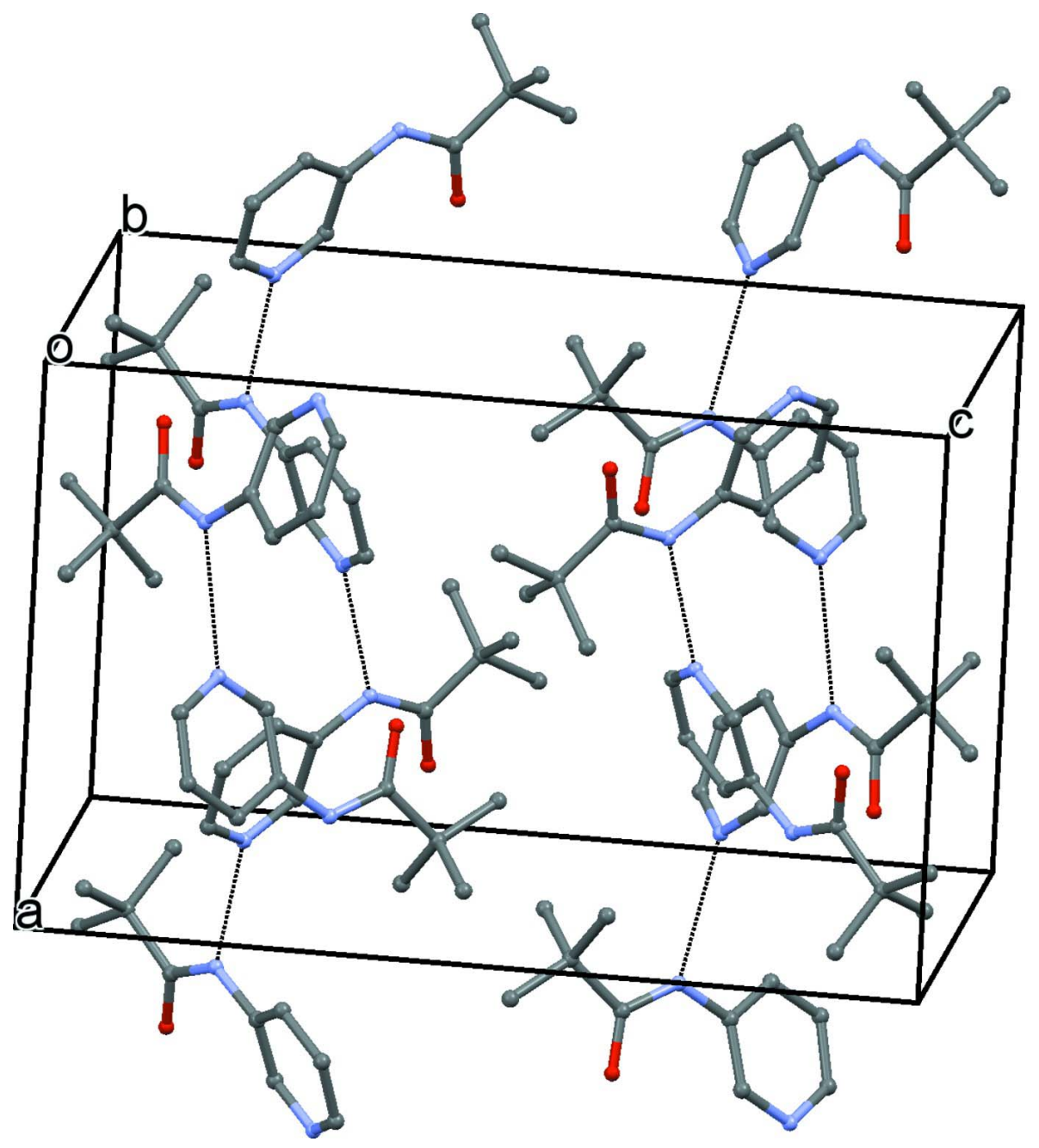

Figure 2

Crystal packing of the title compound, viewed along the $b$ axis, with the $\mathrm{N}-\mathrm{H} \cdots \mathrm{N}$ interactions shown as dashed lines (see Table 1 for details). The minor component of the disordered $t$-butyl group has been omitted for clarity.

\section{2,2-Dimethyl-N-(pyridin-3-yl)propanamide}

\section{Crystal data}

$\mathrm{C}_{10} \mathrm{H}_{14} \mathrm{~N}_{2} \mathrm{O}$

$M_{r}=178.23$

Orthorhombic, $\mathrm{Pbca}$

$a=11.2453$ (3) $\AA$

$b=10.5272(3) \AA$

$c=17.5339$ (6) $\AA$

$V=2075.69(11) \AA^{3}$

$Z=8$

$F(000)=768$
$D_{\mathrm{x}}=1.141 \mathrm{Mg} \mathrm{m}^{-3}$

$\mathrm{Cu} K \alpha$ radiation, $\lambda=1.54184 \AA$

Cell parameters from 3061 reflections

$\theta=5.0-73.4^{\circ}$

$\mu=0.60 \mathrm{~mm}^{-1}$

$T=293 \mathrm{~K}$

Plate, colourless

$0.23 \times 0.19 \times 0.06 \mathrm{~mm}$ 


\section{Data collection}

Agilent SuperNova (Dual, $\mathrm{Cu}$ at zero, Atlas) diffractometer

Radiation source: sealed X-ray tube, SuperNova

$\mathrm{C} \mathrm{Cu}) \mathrm{X}$-ray Source

Mirror monochromator

$\omega$ scans

Absorption correction: multi-scan

(CrysAlis PRO; Agilent, 2014)

$T_{\min }=0.840, T_{\max }=1.000$

\section{Refinement}

Refinement on $F^{2}$

Least-squares matrix: full

$R\left[F^{2}>2 \sigma\left(F^{2}\right)\right]=0.041$

$w R\left(F^{2}\right)=0.127$

$S=1.05$

2065 reflections

153 parameters

114 restraints

Hydrogen site location: inferred from

neighbouring sites
7164 measured reflections

2065 independent reflections

1722 reflections with $I>2 \sigma(I)$

$R_{\text {int }}=0.017$

$\theta_{\max }=73.8^{\circ}, \theta_{\min }=5.1^{\circ}$

$h=-14 \rightarrow 8$

$k=-13 \rightarrow 12$

$l=-20 \rightarrow 21$
$\mathrm{H}$-atom parameters constrained

$w=1 /\left[\sigma^{2}\left(F_{\mathrm{o}}^{2}\right)+(0.0688 P)^{2}+0.2387 P\right]$

where $P=\left(F_{\mathrm{o}}{ }^{2}+2 F_{\mathrm{c}}{ }^{2}\right) / 3$

$(\Delta / \sigma)_{\max }<0.001$

$\Delta \rho_{\max }=0.19$ e $\AA^{-3}$

$\Delta \rho_{\min }=-0.16$ e $\AA^{-3}$

Extinction correction: SHELXL2013 (Sheldrick, 2015), $\mathrm{Fc}^{*}=\mathrm{kFc}\left[1+0.001 \mathrm{xFc}^{2} \lambda^{3} / \sin (2 \theta)\right]^{-1 / 4}$

Extinction coefficient: 0.0016 (4)

\section{Special details}

Geometry. All e.s.d.'s (except the e.s.d. in the dihedral angle between two 1.s. planes) are estimated using the full covariance matrix. The cell e.s.d.'s are taken into account individually in the estimation of e.s.d.'s in distances, angles and torsion angles; correlations between e.s.d.'s in cell parameters are only used when they are defined by crystal symmetry. An approximate (isotropic) treatment of cell e.s.d.'s is used for estimating e.s.d.'s involving 1.s. planes.

Fractional atomic coordinates and isotropic or equivalent isotropic displacement parameters $\left(\AA^{2}\right)$

\begin{tabular}{llllll}
\hline & $x$ & $y$ & $z$ & $U_{\text {iso }} / U_{\text {eq }}$ & Occ. $(<1)$ \\
\hline C1 & $0.72804(10)$ & $0.10403(12)$ & $0.27358(7)$ & $0.0490(3)$ & \\
C2 & $0.61380(11)$ & $0.14128(14)$ & $0.25328(8)$ & $0.0585(4)$ & \\
H2 & 0.5822 & 0.2140 & 0.2756 & $0.070^{*}$ & \\
C3 & $0.59204(13)$ & $-0.02662(15)$ & $0.17123(8)$ & $0.0663(4)$ & \\
H3 & 0.5452 & -0.0722 & 0.1371 & $0.080^{*}$ & \\
C4 & $0.70473(13)$ & $-0.06915(16)$ & $0.18680(9)$ & $0.0702(4)$ & \\
H4 & 0.7344 & -0.1414 & 0.1630 & $0.084^{*}$ & \\
C5 & $0.77323(12)$ & $-0.00301(14)$ & $0.23832(8)$ & $0.0619(4)$ & \\
H5 & 0.8499 & -0.0303 & 0.2494 & $0.074^{*}$ & \\
C6 & $0.76123(12)$ & $0.24951(14)$ & $0.38234(8)$ & $0.0585(3)$ & \\
C7 & $0.85629(13)$ & $0.29549(15)$ & $0.43884(9)$ & $0.0671(4)$ & \\
C8 & $0.9594(3)$ & $0.3605(4)$ & $0.3935(2)$ & $0.0798(10)$ & $0.758(12)$ \\
H8A & 0.9277 & 0.4278 & 0.3627 & $0.120^{*}$ & $0.758(12)$ \\
H8B & 0.9974 & 0.2988 & 0.3613 & $0.120^{*}$ & $0.758(12)$ \\
H8C & 1.0165 & 0.3947 & 0.4287 & $0.120^{*}$ & $0.758(12)$ \\
C9 & $0.9077(5)$ & $0.1835(4)$ & $0.4813(4)$ & $0.0838(11)$ & $0.758(12)$ \\
H9A & 0.9680 & 0.2125 & 0.5159 & $0.126^{*}$ & $0.758(12)$ \\
H9B & 0.9421 & 0.1250 & 0.4455 & $0.126^{*}$ & $0.758(12)$ \\
H9C & 0.8458 & 0.1417 & 0.5094 & $0.126^{*}$ & $0.758(12)$
\end{tabular}




$\begin{array}{llllll}\text { C10 } & 0.8023(4) & 0.3941(7) & 0.4916(4) & 0.1205(18) & 0.758(12) \\ \text { H10A } & 0.7365 & 0.3574 & 0.5186 & 0.181^{*} & 0.758(12) \\ \text { H10B } & 0.7751 & 0.4653 & 0.4621 & 0.181^{*} & 0.758(12) \\ \text { H10C } & 0.8613 & 0.4222 & 0.5274 & 0.181^{*} & 0.758(12) \\ \text { C8A } & 0.9208(16) & 0.4067(14) & 0.4095(8) & 0.108(4) & 0.242(12) \\ \text { H8D } & 0.9534 & 0.3873 & 0.3602 & 0.162^{*} & 0.242(12) \\ \text { H8E } & 0.9841 & 0.4281 & 0.4439 & 0.162^{*} & 0.242(12) \\ \text { H8F } & 0.8672 & 0.4772 & 0.4052 & 0.162^{*} & 0.242(12) \\ \text { C9A } & 0.9385(16) & 0.1880(15) & 0.4658(11) & 0.089(4) & 0.242(12) \\ \text { H9D } & 0.8920 & 0.1137 & 0.4766 & 0.133^{*} & 0.242(12) \\ \text { H9E } & 0.9796 & 0.2142 & 0.5111 & 0.133^{*} & 0.242(12) \\ \text { H9F } & 0.9953 & 0.1690 & 0.4265 & 0.133^{*} & 0.242(12) \\ \text { C10A } & 0.7832(11) & 0.3342(17) & 0.5131(6) & 0.098(4) & 0.242(12) \\ \text { H10D } & 0.7211 & 0.3924 & 0.4993 & 0.147^{*} & 0.242(12) \\ \text { H10E } & 0.8355 & 0.3739 & 0.5491 & 0.147^{*} & 0.242(12) \\ \text { H10F } & 0.7488 & 0.2595 & 0.5355 & 0.147^{*} & 0.242(12) \\ \text { N1 } & 0.79882(8) & 0.16796(11) & 0.32735(6) & 0.0543(3) & \\ \text { H1 } & 0.8741 & 0.1539 & 0.3252 & 0.065^{*} & \\ \text { N2 } & 0.54740(9) & 0.07752(13) & 0.20320(7) & 0.0650(3) & \\ \text { O1 } & 0.65818(9) & 0.28385(14) & 0.38687(7) & 0.0885(4) & \end{array}$

Atomic displacement parameters $\left(\AA^{2}\right)$

\begin{tabular}{lllllll}
\hline & $U^{11}$ & $U^{22}$ & $U^{33}$ & $U^{12}$ & $U^{13}$ & $U^{23}$ \\
\hline C1 & $0.0369(6)$ & $0.0624(7)$ & $0.0478(6)$ & $0.0007(5)$ & $0.0006(5)$ & $0.0031(5)$ \\
C2 & $0.0400(6)$ & $0.0715(8)$ & $0.0640(7)$ & $0.0064(6)$ & $-0.0046(5)$ & $-0.0063(6)$ \\
C3 & $0.0529(7)$ & $0.0841(9)$ & $0.0617(8)$ & $-0.0037(7)$ & $-0.0065(6)$ & $-0.0111(7)$ \\
C4 & $0.0630(9)$ & $0.0778(9)$ & $0.0697(9)$ & $0.0116(7)$ & $-0.0067(7)$ & $-0.0172(7)$ \\
C5 & $0.0450(7)$ & $0.0766(8)$ & $0.0641(8)$ & $0.0130(6)$ & $-0.0051(6)$ & $-0.0078(6)$ \\
C6 & $0.0461(7)$ & $0.0715(8)$ & $0.0579(7)$ & $0.0057(6)$ & $-0.0020(6)$ & $-0.0039(6)$ \\
C7 & $0.0620(8)$ & $0.0739(8)$ & $0.0653(8)$ & $-0.0024(7)$ & $-0.0092(6)$ & $-0.0103(7)$ \\
C8 & $0.0716(17)$ & $0.0753(17)$ & $0.0926(19)$ & $-0.0182(13)$ & $-0.0135(13)$ & $0.0017(14)$ \\
C9 & $0.089(3)$ & $0.101(2)$ & $0.062(2)$ & $-0.0202(16)$ & $-0.0235(18)$ & $0.0146(16)$ \\
C10 & $0.098(2)$ & $0.134(4)$ & $0.129(4)$ & $-0.002(3)$ & $-0.004(2)$ & $-0.073(3)$ \\
C8A & $0.129(8)$ & $0.107(7)$ & $0.087(6)$ & $-0.026(6)$ & $-0.027(6)$ & $0.000(6)$ \\
C9A & $0.080(7)$ & $0.116(7)$ & $0.071(7)$ & $0.008(6)$ & $-0.023(5)$ & $-0.011(5)$ \\
C10A & $0.102(6)$ & $0.115(8)$ & $0.076(5)$ & $0.013(6)$ & $-0.026(4)$ & $-0.046(5)$ \\
N1 & $0.0341(5)$ & $0.0708(7)$ & $0.0581(6)$ & $0.0041(4)$ & $-0.0035(4)$ & $-0.0056(5)$ \\
N2 & $0.0408(6)$ & $0.0867(8)$ & $0.0676(7)$ & $0.0032(5)$ & $-0.0078(5)$ & $-0.0079(6)$ \\
O1 & $0.0543(6)$ & $0.1273(10)$ & $0.0838(8)$ & $0.0235(6)$ & $-0.0048(5)$ & $-0.0356(7)$ \\
& & & & & & \\
\hline
\end{tabular}

Geometric parameters $\left(\AA,{ }^{\circ}\right)$

\begin{tabular}{llll}
\hline $\mathrm{C} 1-\mathrm{C} 5$ & $1.3820(19)$ & $\mathrm{C} 8-\mathrm{H} 8 \mathrm{~A}$ & 0.9600 \\
$\mathrm{C} 1-\mathrm{C} 2$ & $1.3895(17)$ & $\mathrm{C} 8-\mathrm{H} 8 \mathrm{~B}$ & 0.9600 \\
$\mathrm{C} 1-\mathrm{N} 1$ & $1.4054(16)$ & $\mathrm{C} 8-\mathrm{H} 8 \mathrm{C}$ & 0.9600 \\
$\mathrm{C} 2-\mathrm{N} 2$ & $1.3339(18)$ & $\mathrm{C} 9-\mathrm{H} 9 \mathrm{~A}$ & 0.9600 \\
$\mathrm{C} 2-\mathrm{H} 2$ & 0.9300 & $\mathrm{C} 9-\mathrm{H} 9 \mathrm{~B}$ & 0.9600
\end{tabular}




\begin{tabular}{|c|c|c|c|}
\hline $\mathrm{C} 3-\mathrm{N} 2$ & $1.3297(19)$ & $\mathrm{C} 9-\mathrm{H} 9 \mathrm{C}$ & 0.9600 \\
\hline $\mathrm{C} 3-\mathrm{C} 4$ & $1.372(2)$ & $\mathrm{C} 10-\mathrm{H} 10 \mathrm{~A}$ & 0.9600 \\
\hline $\mathrm{C} 3-\mathrm{H} 3$ & 0.9300 & $\mathrm{C} 10-\mathrm{H} 10 \mathrm{~B}$ & 0.9600 \\
\hline $\mathrm{C} 4-\mathrm{C} 5$ & $1.376(2)$ & $\mathrm{C} 10-\mathrm{H} 10 \mathrm{C}$ & 0.9600 \\
\hline $\mathrm{C} 4-\mathrm{H} 4$ & 0.9300 & $\mathrm{C} 8 \mathrm{~A}-\mathrm{H} 8 \mathrm{D}$ & 0.9600 \\
\hline $\mathrm{C} 5-\mathrm{H} 5$ & 0.9300 & $\mathrm{C} 8 \mathrm{~A}-\mathrm{H} 8 \mathrm{E}$ & 0.9600 \\
\hline $\mathrm{C} 6-\mathrm{O} 1$ & $1.2165(17)$ & $\mathrm{C} 8 \mathrm{~A}-\mathrm{H} 8 \mathrm{~F}$ & 0.9600 \\
\hline $\mathrm{C} 6-\mathrm{N} 1$ & $1.3585(17)$ & C9A-H9D & 0.9600 \\
\hline $\mathrm{C} 6-\mathrm{C} 7$ & $1.5356(19)$ & C9A-H9E & 0.9600 \\
\hline $\mathrm{C} 7-\mathrm{C} 8 \mathrm{~A}$ & $1.470(7)$ & C9A-H9F & 0.9600 \\
\hline $\mathrm{C} 7-\mathrm{C} 9$ & $1.510(4)$ & $\mathrm{C} 10 \mathrm{~A}-\mathrm{H} 10 \mathrm{D}$ & 0.9600 \\
\hline $\mathrm{C} 7-\mathrm{C} 10$ & $1.517(4)$ & $\mathrm{C} 10 \mathrm{~A}-\mathrm{H} 10 \mathrm{E}$ & 0.9600 \\
\hline $\mathrm{C} 7-\mathrm{C} 9 \mathrm{~A}$ & $1.536(8)$ & $\mathrm{C} 10 \mathrm{~A}-\mathrm{H} 10 \mathrm{~F}$ & 0.9600 \\
\hline $\mathrm{C} 7-\mathrm{C} 8$ & $1.563(3)$ & $\mathrm{N} 1-\mathrm{H} 1$ & 0.8600 \\
\hline $\mathrm{C} 7-\mathrm{C} 10 \mathrm{~A}$ & $1.593(7)$ & & \\
\hline $\mathrm{C} 5-\mathrm{C} 1-\mathrm{C} 2$ & $117.09(12)$ & $\mathrm{H} 8 \mathrm{~B}-\mathrm{C} 8-\mathrm{H} 8 \mathrm{C}$ & 109.5 \\
\hline $\mathrm{C} 5-\mathrm{C} 1-\mathrm{N} 1$ & $118.83(10)$ & $\mathrm{C} 7-\mathrm{C} 9-\mathrm{H} 9 \mathrm{~A}$ & 109.5 \\
\hline $\mathrm{C} 2-\mathrm{C} 1-\mathrm{N} 1$ & $124.07(11)$ & C7-C9-H9B & 109.5 \\
\hline $\mathrm{N} 2-\mathrm{C} 2-\mathrm{C} 1$ & $122.97(12)$ & $\mathrm{H} 9 \mathrm{~A}-\mathrm{C} 9-\mathrm{H} 9 \mathrm{~B}$ & 109.5 \\
\hline $\mathrm{N} 2-\mathrm{C} 2-\mathrm{H} 2$ & 118.5 & C7- $99-\mathrm{H} 9 \mathrm{C}$ & 109.5 \\
\hline $\mathrm{C} 1-\mathrm{C} 2-\mathrm{H} 2$ & 118.5 & $\mathrm{H} 9 \mathrm{~A}-\mathrm{C} 9-\mathrm{H} 9 \mathrm{C}$ & 109.5 \\
\hline $\mathrm{N} 2-\mathrm{C} 3-\mathrm{C} 4$ & $122.28(13)$ & $\mathrm{H} 9 \mathrm{~B}-\mathrm{C} 9-\mathrm{H} 9 \mathrm{C}$ & 109.5 \\
\hline $\mathrm{N} 2-\mathrm{C} 3-\mathrm{H} 3$ & 118.9 & $\mathrm{C} 7-\mathrm{C} 10-\mathrm{H} 10 \mathrm{~A}$ & 109.5 \\
\hline $\mathrm{C} 4-\mathrm{C} 3-\mathrm{H} 3$ & 118.9 & $\mathrm{C} 7-\mathrm{C} 10-\mathrm{H} 10 \mathrm{~B}$ & 109.5 \\
\hline $\mathrm{C} 3-\mathrm{C} 4-\mathrm{C} 5$ & $118.86(14)$ & $\mathrm{H} 10 \mathrm{~A}-\mathrm{C} 10-\mathrm{H} 10 \mathrm{~B}$ & 109.5 \\
\hline $\mathrm{C} 3-\mathrm{C} 4-\mathrm{H} 4$ & 120.6 & $\mathrm{C} 7-\mathrm{C} 10-\mathrm{H} 10 \mathrm{C}$ & 109.5 \\
\hline $\mathrm{C} 5-\mathrm{C} 4-\mathrm{H} 4$ & 120.6 & $\mathrm{H} 10 \mathrm{~A}-\mathrm{C} 10-\mathrm{H} 10 \mathrm{C}$ & 109.5 \\
\hline $\mathrm{C} 4-\mathrm{C} 5-\mathrm{C} 1$ & $120.02(12)$ & $\mathrm{H} 10 \mathrm{~B}-\mathrm{C} 10-\mathrm{H} 10 \mathrm{C}$ & 109.5 \\
\hline $\mathrm{C} 4-\mathrm{C} 5-\mathrm{H} 5$ & 120.0 & $\mathrm{C} 7-\mathrm{C} 8 \mathrm{~A}-\mathrm{H} 8 \mathrm{D}$ & 109.5 \\
\hline $\mathrm{C} 1-\mathrm{C} 5-\mathrm{H} 5$ & 120.0 & $\mathrm{C} 7-\mathrm{C} 8 \mathrm{~A}-\mathrm{H} 8 \mathrm{E}$ & 109.5 \\
\hline $\mathrm{O} 1-\mathrm{C} 6-\mathrm{N} 1$ & $122.04(13)$ & $\mathrm{H} 8 \mathrm{D}-\mathrm{C} 8 \mathrm{~A}-\mathrm{H} 8 \mathrm{E}$ & 109.5 \\
\hline $\mathrm{O} 1-\mathrm{C} 6-\mathrm{C} 7$ & $121.83(13)$ & $\mathrm{C} 7-\mathrm{C} 8 \mathrm{~A}-\mathrm{H} 8 \mathrm{~F}$ & 109.5 \\
\hline $\mathrm{N} 1-\mathrm{C} 6-\mathrm{C} 7$ & $116.13(11)$ & $\mathrm{H} 8 \mathrm{D}-\mathrm{C} 8 \mathrm{~A}-\mathrm{H} 8 \mathrm{~F}$ & 109.5 \\
\hline $\mathrm{C} 9-\mathrm{C} 7-\mathrm{C} 10$ & $112.8(3)$ & $\mathrm{H} 8 \mathrm{E}-\mathrm{C} 8 \mathrm{~A}-\mathrm{H} 8 \mathrm{~F}$ & 109.5 \\
\hline $\mathrm{C} 8 \mathrm{~A}-\mathrm{C} 7-\mathrm{C} 6$ & $111.6(5)$ & C7-C9A-H9D & 109.5 \\
\hline $\mathrm{C} 9-\mathrm{C} 7-\mathrm{C} 6$ & $109.8(3)$ & C7-C9A-H9E & 109.5 \\
\hline $\mathrm{C} 10-\mathrm{C} 7-\mathrm{C} 6$ & $109.3(2)$ & $\mathrm{H} 9 \mathrm{D}-\mathrm{C} 9 \mathrm{~A}-\mathrm{H} 9 \mathrm{E}$ & 109.5 \\
\hline $\mathrm{C} 8 \mathrm{~A}-\mathrm{C} 7-\mathrm{C} 9 \mathrm{~A}$ & $113.4(7)$ & $\mathrm{C} 7-\mathrm{C} 9 \mathrm{~A}-\mathrm{H} 9 \mathrm{~F}$ & 109.5 \\
\hline $\mathrm{C} 6-\mathrm{C} 7-\mathrm{C} 9 \mathrm{~A}$ & $112.7(9)$ & $\mathrm{H} 9 \mathrm{D}-\mathrm{C} 9 \mathrm{~A}-\mathrm{H} 9 \mathrm{~F}$ & 109.5 \\
\hline $\mathrm{C} 9-\mathrm{C} 7-\mathrm{C} 8$ & $107.9(2)$ & $\mathrm{H} 9 \mathrm{E}-\mathrm{C} 9 \mathrm{~A}-\mathrm{H} 9 \mathrm{~F}$ & 109.5 \\
\hline $\mathrm{C} 10-\mathrm{C} 7-\mathrm{C} 8$ & $107.9(2)$ & $\mathrm{C} 7-\mathrm{C} 10 \mathrm{~A}-\mathrm{H} 10 \mathrm{D}$ & 109.5 \\
\hline $\mathrm{C} 6-\mathrm{C} 7-\mathrm{C} 8$ & $109.05(17)$ & $\mathrm{C} 7-\mathrm{C} 10 \mathrm{~A}-\mathrm{H} 10 \mathrm{E}$ & 109.5 \\
\hline $\mathrm{C} 8 \mathrm{~A}-\mathrm{C} 7-\mathrm{C} 10 \mathrm{~A}$ & $109.7(5)$ & $\mathrm{H} 10 \mathrm{D}-\mathrm{C} 10 \mathrm{~A}-\mathrm{H} 10 \mathrm{E}$ & 109.5 \\
\hline $\mathrm{C} 6-\mathrm{C} 7-\mathrm{C} 10 \mathrm{~A}$ & $104.4(5)$ & $\mathrm{C} 7-\mathrm{C} 10 \mathrm{~A}-\mathrm{H} 10 \mathrm{~F}$ & 109.5 \\
\hline $\mathrm{C} 9 \mathrm{~A}-\mathrm{C} 7-\mathrm{C} 10 \mathrm{~A}$ & $104.3(6)$ & $\mathrm{H} 10 \mathrm{D}-\mathrm{C} 10 \mathrm{~A}-\mathrm{H} 10 \mathrm{~F}$ & 109.5 \\
\hline $\mathrm{C} 7-\mathrm{C} 8-\mathrm{H} 8 \mathrm{~A}$ & 109.5 & $\mathrm{H} 10 \mathrm{E}-\mathrm{C} 10 \mathrm{~A}-\mathrm{H} 10 \mathrm{~F}$ & 109.5 \\
\hline $\mathrm{C} 7-\mathrm{C} 8-\mathrm{H} 8 \mathrm{~B}$ & 109.5 & $\mathrm{C} 6-\mathrm{N} 1-\mathrm{C} 1$ & $127.05(10)$ \\
\hline
\end{tabular}




$\begin{array}{llll}\mathrm{H} 8 \mathrm{~A}-\mathrm{C} 8-\mathrm{H} 8 \mathrm{~B} & 109.5 & \mathrm{C} 6-\mathrm{N} 1-\mathrm{H} 1 & 116.5 \\ \mathrm{C} 7-\mathrm{C} 8-\mathrm{H} 8 \mathrm{C} & 109.5 & \mathrm{C} 1-\mathrm{N} 1-\mathrm{H} 1 & 116.5 \\ \mathrm{H} 8 \mathrm{~A}-\mathrm{C} 8-\mathrm{H} 8 \mathrm{C} & 109.5 & \mathrm{C} 3-\mathrm{N} 2-\mathrm{C} 2 & 118.76(11)\end{array}$

Hydrogen-bond geometry $\left(A,{ }^{\circ}\right)$

\begin{tabular}{lllll}
\hline$D-\mathrm{H} \cdots A$ & $D-\mathrm{H}$ & $\mathrm{H} \cdots A$ & $D \cdots A$ & $D-\mathrm{H} \cdots A$ \\
\hline $\mathrm{C} 2-\mathrm{H} 2 \cdots \mathrm{O} 1$ & 0.93 & 2.25 & $2.8263(18)$ & 119 \\
$\mathrm{~N} 1-\mathrm{H} 1 \cdots \mathrm{N} 2^{\mathrm{i}}$ & 0.86 & 2.17 & $3.0012(15)$ & 164 \\
\hline
\end{tabular}

Symmetry code: (i) $x+1 / 2, y,-z+1 / 2$. 